\title{
Linfoma NK/T extranodal diagnosticado como celulitis orbitaria refractaria a tratamiento
}

\section{Extranodal NK/T lymphoma diagnosed as refractory orbital cellulitis}

\author{
Karyn DianaTito-Eusebio', Dayana Carolina \\ Gil-Clares², Allison Eliana Yanire Juárez- \\ Pimente $^{3}$
}

Tito-EusebioK, Gil-Clares D, Juárez- Pimentel A. Linfoma NK/T extranodal diagnosticado como celulitis orbitaria refractaria a tratamiento. Rev Soc Peru Med Interna. 202 I;34(4): I90-195. DOI: https://doi.org/10.36393/spmi.v34i4.637

\begin{abstract}
RESUMEN
Varón de 60 años, con antecedente familiar de hermano fallecido con diagnóstico de linfoma de células Natural Killer/T extranodales (LNKTE) tipo nasal, presentó un cuadro clínico compatible con celulitis orbitaria izquierda y, posteriormente, deterioro del estado general e insuficiencia hepática. Sin adecuada respuesta a los antibióticos, la biopsia del párpado reveló un LNKTE. Recibió tratamiento con quimioterapia y falleció un mes después del diagnóstico.
\end{abstract}

Palabras claves: Linfoma, extranodal, células Natural Killer/T, celulitis orbitaria, insuficiencia hepática.

\section{ABSTRACT}

A 60-year-old male, with a family history of a deceased brother diagnosed with nasal-type Natural Killer/T cell lymphoma (LNKTE), presented a clinical picture compatible with left orbital cellulitis and, subsequently, deterioration of general condition and liver failure. Without adequate response to antibiotics, eyelid biopsy revealed an LNKTE. He was treated with chemotherapy and died a month after diagnosis.

Keywords: Lymphoma, extranodal, Natural Killer/T cells, orbital cellulite, liver failure.

\section{INTRODUCCIÓN}

Los linfomas de células Natural Killer/T extranodales (LNKTE) son un tipo infrecuente de linfoma no Hodgkin donde la mayoría son de tipo nasal, las cuales comprenden

Médica residente de medicina familiar. Universidad Ricardo Palma. Centro de Salud I-3 Chacarilla de Otero, San Juan de Lurigancho, Lima, Perú.

2 Médica residente de medicina interna. Universidad Ricardo Palma. Hospital II Luis Negreiros Vega-EsSalud, Callao, Perú.

3 Médica-cirujano. Egresada de la Universidad Nacional Mayor de San Marcos.Villa Panamericana-EsSalud.Villa El Salvador, Lima, Perú. la vía aérea superior, cavidad nasal, senos paranasales, laringe y, muy infrecuentemente, las órbitas oculares. ${ }^{1,2}$ Esta patología es de difícil diagnóstico. Se presenta inicialmente como inflamación indolora de los párpados en un paciente con antecedente de sinusitis crónica o recurrente, siendo el edema y ptosis las manifestaciones más comunes. ${ }^{3-5}$ Por ello suele ser diagnosticado como celulitis orbitaria en el $50 \%$ de los casos. . $^{4-8}$

El diagnóstico se confirma mediante inmunohistoquímica, que es el gold estándar. En la patología se evidencia extensas áreas de necrosis y abundante infiltrado inflamatorio que puede ocultar células neoplásicas. Por otro 
lado, en la inmunohistoquímica se evidencia angioinvasión, angiodestrucción y crecimiento angiocéntrico, positividad para CD2, CD16, CD56, CD57 e hibridización in situ para ARN del virus Epstein Barr (VEB), con el cual suele estar asociado. ${ }^{4,6}$

Los pacientes con LNKTE localizado requieren diagnóstico temprano, ser tratados con terapia combinada (CMT) con radioterapia y quimioterapia; sin embargo, el pronóstico es usualmente malo debido al diagnóstico tardío y a la recurrencia o refractariedad al tratamiento. ${ }^{3}$

\section{PRESENTACIÓN DEL CASO}

Varón de 60 años quien fue referido al servicio de medicina interna por celulitis periorbitaria izquierda de aproximadamente siete meses de enfermedad refractaria a tratamientos antibióticos y asociada a insuficiencia hepática.

El paciente debutó con lagrimeo, sensación de quemazón del ojo izquierdo, seguido de enrojecimiento, edema, ptosis palpebral y signos clínicos de celulitis local (Figura 1). Posteriormente se agregó insuficiencia hepática, bicitopenia y síndrome febril. Como antecedente, septoplastía hace cuatro meses debido a sinusitis crónica y, como antecedente familiar, un hermano fallecido por LNKTE nasal.

Asimismo, una tomografía computarizada (TC) sin contraste de senos paranasales a seis meses del inicio de los síntomas solo evidenció edema en la región palpebral izquierda (Figuras 2A y 2B).

A su ingreso, en la región palpebral superior del lado izquierdo se halló una neoformación granulomatosa

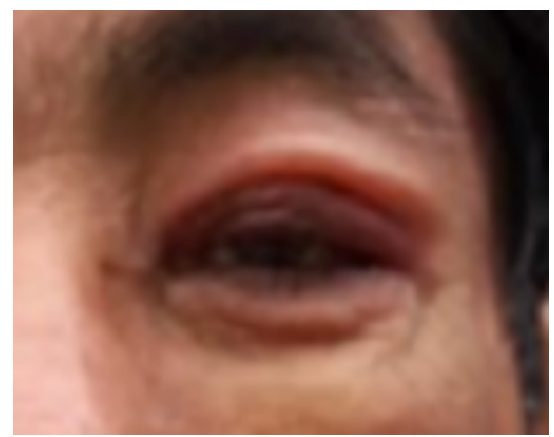

Figura I. Edema y eritema del párpado superior izquierdo.

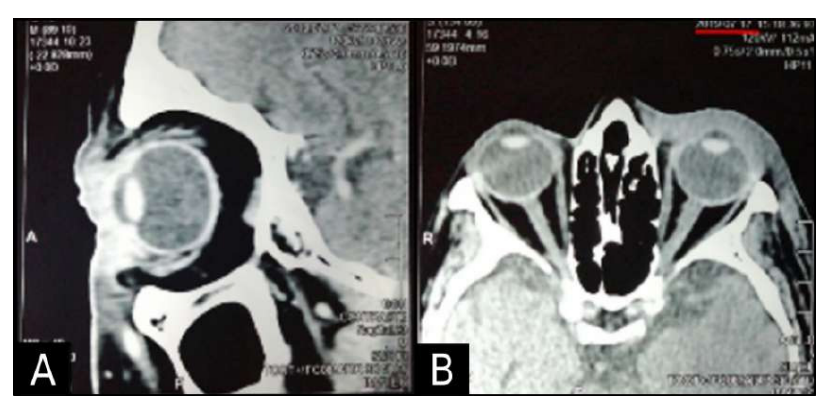

Figura 2. Tomografías sin contraste. (A) plano sagital, aumento de intensidad en región palpebral superior con integridad del globo ocular; (B) plano axial, edema de la región palpebral izquierda con integridad de globo ocular. costrosa, con secreción mucopurulenta circundante, sin afectación de la agudeza visual ni movimientos oculares (Figura 3). Edema del miembro superior derecho con algunas lesiones ulceradas y costrosas ovales, bien delimitadas, no mayores de $3 \mathrm{~cm}$ de diámetro, indoloras; $\mathrm{y}$, una lesión con centro necrótico en la pierna derecha (Figuras 4 y 5). No se palparon adenomegalias. Pulmones y cardiovascular normales. Abdomen, blando, con hepatomegalia.

Con el diagnóstico presuntivo de celulitis orbitaria izquierda con probable absceso periorbitario se inició tratamiento con vancomicina y piperacilina/tazobactam; sin embargo, no se observó mejoría. Los exámenes de biometría hemática y química sanguínea concluyeron pancitopenia, azoemia, alteración del perfil hepático y de coagulación.

Se realizaron tomografías de tórax y abdomen sin contraste que evidenciaron hepatomegalia, no adenopatías ni otra información contributoria. Los estudios serológicos para VIH, hepatitis B, hepatitis C y HTLV1 fueron negativos. Cultivos, Ziehl-Neelsen de secreciones y esputo negativos. Citología y citoquímica de líquido cefalorraquídeo sin alteraciones. No se encontró evidencia que sustente la falla hepática.

El paciente evolucionó desfavorablemente con persistencia de falla hepática aguda intermitente y pancitopenia, por lo que requirió transfusiones sanguíneas frecuentes además de rotación a antibióticos no-hepatotóxicos en dosis ajustadas y finalmente suspensión total de antibióticos.

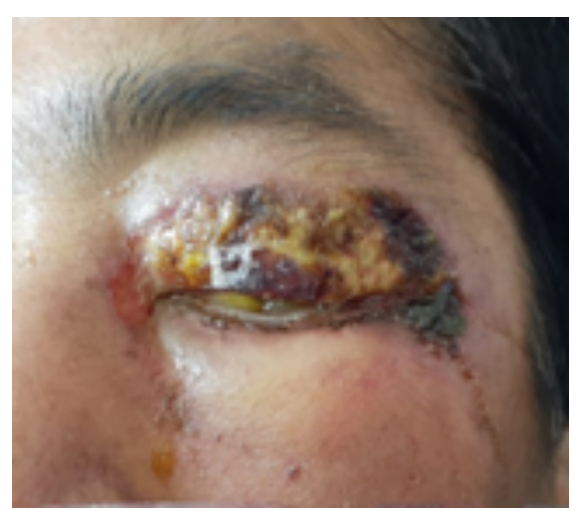

Figura 3. Lesión costrosa y con secreción purulenta del párpado superior.

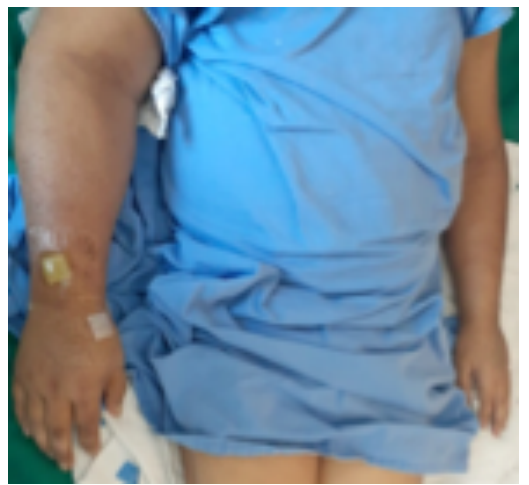

Figura 4. Edema del miembro superior derecho y lesiones ulcerosas a nivel de la muñeca. 


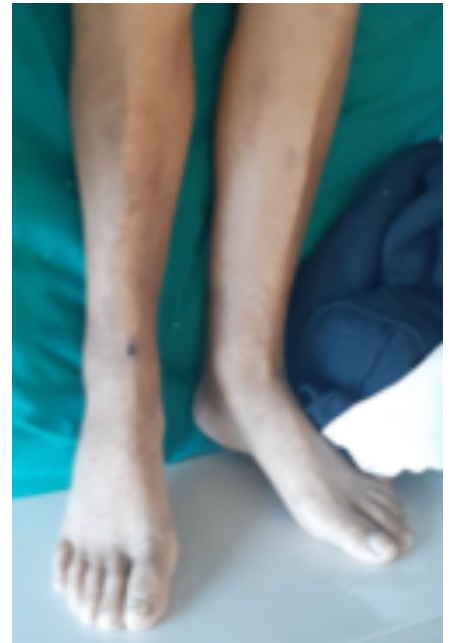

Figura 5. Lesión ulcerosa y costrosa, seca y aislada en la región pretibial derecha.

Se realizó una biopsia de piel de antebrazo que reportó dermis con infiltrado inflamatorio mixto perivascular, a predominio linfoplasmocitario, sin evidencia de células neoplásicas. La biopsia de piel de párpado superior evidenció necrosis de epidermis, infiltración plasmocitaria mas no se encontró granulomas, las tinciones de PAS y Ziehl-Neelsen resultaron negativas (Figura 6). Se repitió la biopsia del párpado para estudios histológicos e inmunohistoquímica cuyos hallazgos fueron: VEB-, CD20-, CD3+, CD45ro-, CD8+ y ki67+ 67\%. Los resultados fueron reportados por el servicio de patología como linfoma no Hodgkin fenotipo $\mathrm{T}$ variante NK.

Con el diagnóstico de LNKTE primario de órbita estadío clínico IIE se inició tratamiento con tres cursos de quimioterapia con esquema SMILE (dexametasona, metotrexato, ifosfamida, L-asparginasa y etopósido) más FEC (filgastrim), antibioticoterapia con linezolid y piperacilina/tazobactam. El paciente cursó con evolución tórpida y falleció por falla orgánica múltiple.

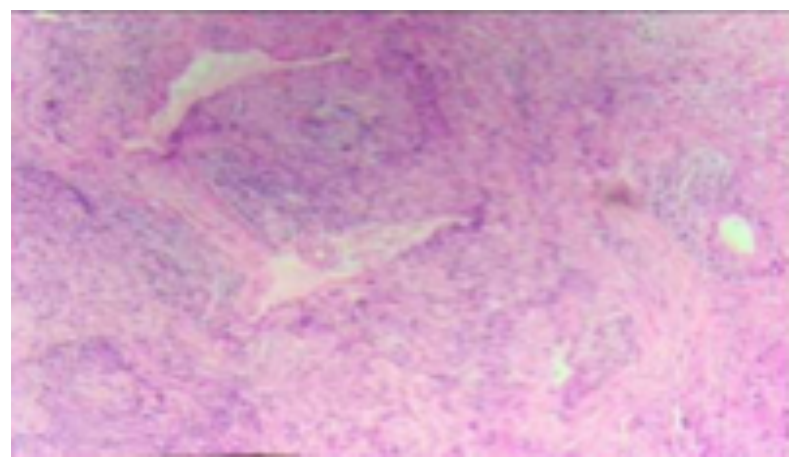

Figura 6. H-E. Dermis un denso infiltrado inflamatorio mixto que se extiende hasta el tejido celular subcutáneo, constituido por linfocitos, células plasmáticas y neutrófilos. La mitad de las células son de tamaño mediano $y$ en menor proporción células grandes y pequeñas. Los linfocitos tienen tendencia a invadir y destruir vasos sanguíneos.

\section{DISCUSIÓN}

Los LNKTE involucran mayormente el área nasal y de los senos paranasales de los cuales, el primero representa el $87 \%$ de los LNKTE, seguido de la piel en 5,4\%, tracto gastrointestinal en 3,6\%, tejido celular subcutáneo 3,8\% y testes en $1,8 \%$ de prevalencia. ${ }^{9}$ Los linfomas extranodales orbitales y periorbitales, constituyen el $3 \%$ de tipo B mientras que son excepcionales los de tipo NK/T. ${ }^{2}$ Los LNKTE orbitales y periorbitales pueden ser de tipo primario o secundario al LNKTE nasal $u$ otras partes del cuerpo..$^{10}$ Ante la presencia de LNKTE extranasales, es relevante descartar la extensión del tipo nasal primario. ${ }^{11}$ Los LNKTE ocular asociados a piel son muy raros y de mal pronóstico, y es más frecuente que sea secundario al tipo nasal. ${ }^{12}$

En el reporte del caso, el paciente con antecedente de sinusitis crónica, no evidenció clínica de patología nasal durante su estancia hospitalaria, además y no se demostró lesiones erosivas en la región nasal ni senos paranasales, por lo cual su abordaje se centró en las lesiones del párpado superior y, posteriormente, en las lesiones ulcerativas de piel.

La población afectada por linfomas NK/T es mayor en Asia y Sudamérica con incidencias del 5,2\% y 3\%, respectivamente, en comparación a Europa y Norteamérica cuyas incidencias son del $0,3 \% .^{3}$ El grupo etario más afectado es el masculino respecto al femenino, a razón de $2: 1$, y suele aparecer en la quinta década de vida y se asocia a la infección por VEB. ${ }^{2,13}$ Nuestro paciente se encontraba en el grupo etario y sexo de mayor prevalencia; $y$, por otro lado, tuvo serología negativa para VEB.

En el 2018, se reportó el caso de dos hermanos varones con LNKTE, en China, de los cuales el mayor de 35 años falleció posterior al tratamiento y el hermano menor de 18 años presentó remisión de la enfermedad posterior al tratamiento. Sin embargo, tres años después fue diagnosticado de leucemia mieloide crónica y falleció por complicaciones relacionados al trasplante de médula ósea seis años después del diagnóstico de leucemia. Un hermano en común y los familiares de primer grado no evidenciaron alguna afectación neoplásica hematológica. Se encontró la mutación de la línea germinal del FAM1600AL en los casos de LNKTE nasal familiar, así como en otros cánceres. ${ }^{14}$ Pese a los avances científicos, la predisposición genética como factor de riesgo es desconocida y la presencia de familiares con linfoma NK/T son extremadamente raros. ${ }^{15}$ En el caso que se presenta, el paciente tuvo un hermano menor que fue diagnosticado con LNKTE nasal, recibió tratamiento y obtuvo mejoría, sin embargo, dos años después falleció por recidiva de la enfermedad.

Los LNKTE oculares suelen ser diagnosticados por primera vez como celulitis orbitaria (10) o pseudotumor que evoluciona rápidamente a ptosis orbitaria. ${ }^{5,10}$ Además, se describen movimientos extraoculares limitados, inflamación orbitaria como uveítis, vitreítis, infiltrados de la órbita y afectación de la retina como lesión macular y desprendimiento de retina. ${ }^{2}$ Se ha descrito LNKTE sino- 
orbital con edema periorbital, diplopía, ptosis, descarga nasal y antecedente de rinosinusitis crónica y biopsia de pólipo nasal sin evidencia de malignidad. ${ }^{16}$ Se registra además lesiones ulcerosas de párpado superior dolorosas o no dolorosas, lesiones limitadas a la región ocular y otras acompañadas de úlceras de piel. ${ }^{10}$ En el presente caso, el paciente presentó signos clínicos de celulitis periorbitaria que evolucionó a lesión costrosa y necrótica sin afectación de la agudeza visual ni de movimientos oculares. Se acompañó de ulceras periféricas de piel que no cicatrizaban y áreas necróticas con signos de flogosis.

En el diagnóstico LNKTE primario de órbita EC IIE, se consideró daño hepático secundario a antibióticos; sin embargo, no se realizó biopsia hepática que confirme metástasis ya que el estudio de imágenes suele ser insuficiente. El daño hepático inducido por antibióticos se presenta en 14-19 casos de 1000000 habitantes y puede presentarse tardíamente, desde semanas, como la amoxicilina, o años, como la nitrofurantoína. Para la mayoría de pacientes, el pronóstico es favorable; sin embargo, ante la ictericia, el 10\% tiene riesgo de muerte o requiere trasplante; la edad avanzada también es un factor de riesgo. ${ }^{17}$ A nuestro paciente, a quien no se le evidenció falla hepática crónica antes, presentó insuficiencia hepática luego de siete meses después del inicio de los síntomas periorbitarios y tres meses después de la administración de diversos antibióticos. El paciente recibió sucesivamente amoxicilina más ácido clavulánico, oxacilina, clindamicina, ceftriaxona, piperacilina-tazobactan, fluconazol, y vancomicina. En el paciente, el edema de extremidades, hipoalbuminemia e ictericia mejoraban momentánea y discretamente con la suspensión de antibióticos, por lo cual se hizo la asociación.

El LNKTE raramente se presenta de forma diseminada, cuando esto ocurre puede infiltrar el hígado causando falla hepática. ${ }^{3}$ Se reportó un caso donde un paciente varón de 33 años recibió quimioterapia debido al diagnóstico de LNKTE nasal, luego presentó falla hepática aguda que se catalogó como secundario a la quimioterapia hasta que el estudio inmunohistoquímico confirmó metástasis hepática. ${ }^{18}$ Asimismo, se publicó un caso de un paciente varón que ingresó por fiebre, malestar general y anorexia, enzimas hepáticas elevadas, hepatoesplenomegalia y linfadenopatía perihepática y paraaórtica; los estudios determinaron LNKTE de tejido hepático; luego, el paciente se complicó con necrosis de sigmoides y afectación ocular, como hipopion y disminución de la agudeza visual, que progresaron pese a quimioterapia y radioterapia; y, finalmente, se agregó un síndrome hemofagocítico. ${ }^{19}$ En nuestro caso, la falla hepática ocurrió meses después de las lesiones oculares y no se evidenció adenomegalias en las tomografías por lo que no se investigó lesiones metastásicas; no obstante, hubo hepatomegalia, hipoalbuminemia, bicitopenia, fiebre y deshidrogenasa láctica elevada.

La tomografía computarizada (TC) y la resonancia magnética nuclear $(\mathrm{RMN})$ realizadas a los pacientes con diagnóstico de linfoma NK/T son inadecuadas ya que no pueden evidenciar las afectaciones subclínicas u ocultas en nariz ni en otros sitios anatómicos; por tanto, los datos no se consideran confiables. ${ }^{2,3}$ Sin embargo, la tomografía por emisión de positrones (PET/TC) es la imagen estándar para el linfoma NK/T; por ende, en los tipos no nasales se debe realizar PET/TC con fluoriodesoxiglucosa F18 con el objetivo de excluir un LNKTE nasal primario oculto. $^{3}$ Nuestro paciente tuvo TC sin contraste en dos oportunidades donde se evidenció signos de sinusitis sin otro dato contributorio.

Las lesiones por LNKTE presentan necrosis y abundante infiltrado inflamatorio, por lo que las biopsias requieren de muestras extensas y profundas en cuña realizados por personal entrenado ${ }^{6,16}$, haciendo uso de endoscopías de senos paranasales ${ }^{8}$, preparación quirúrgica o anestesia general. ${ }^{4}$ Los informes de las muestras a menudo son inconclusos o negativo, por lo que suelen ser sucesivas., ${ }^{4,8,6,16,20}$ Histológicamente se observa infiltrado de células neoplásicas, vistas como linfocitos granulares grandes y angiodestrucción, lo que produce necrosis local. En el caso del paciente, un estudio previo de biopsia de párpado se informó como necrosis e inflamación sin evidencia de neoplasia, por lo que se repitió el procedimiento. La biopsia reveló dermis con áreas de tejido fibroso, hemorragia y necrosis, además focos de infiltrado linfoide de aspecto atípico. El diagnóstico definitivo del LNKTE se obtiene mediante inmunohistoquímica, donde las células del linfoma expresan los inmunofetipos típicos de $\mathrm{C} 2+$, CD3- de superficie, CD56+, moléculas citotóxicas y la infección de las células por el VEB. ${ }^{3,10}$ Una serie de casos de 21 pacientes con LNKTE ocular evidenció en todos CD3 citoplasmático, CD56, moléculas citotóxicas y ADN codificado de VEB.$^{10}$ En el caso clínico expuesto, se realizó inmunohistoquímica para identificación de células atípicas mediante biomarcadores $\mathrm{CD} 3, \mathrm{CD} 8$, los cuales resultaron positivos; CD20, CD45 y VEB negativos, además el índice proliferativo Ki67 positivo en el $67 \%$. Estos hallazgos histológicos e inmunohistoquimicos correspondieron al linfoma no Hodking, fenotipo T, variable NK. Finalmente, en las formas diseminadas de LNKTE se pueden evidenciar hemofagocitosis en el hígado, bazo y médula ósea que producen insuficiencia hepática, hiperferritinemia y pancitopenia. ${ }^{3}$ En nuestro caso, un mielograma previo de cresta iliaca no evidenció hemofagocitosis ni alteraciones de las serie mieloide y linfoide. Por tanto, no se determinó el origen del linfoma mediante estudios especiales de imagen e inmunohistoquímicos ya que no se descarta que fuera nasal tipo primario por los antecedentes patológicos de sinusitis crónica y antecedentes familiares; así mismo no se concluyó el origen del daño hepático mediante estudios inmunohistoquímicos.

En la última década, la radioterapia y la quimioterapia basada en asparginasa y sin antraciclinas mejoraron el pronóstico de la enfermedad. El tratamiento de los LNKTE difiere en la etapa temprana y avanzada: la radioterapia es la piedra angular de intención curativa en la enfermedad localizada pero no cuando está diseminada ya que presenta 
una supervivencia prolongada en estadio temprano. ${ }^{21-23} \mathrm{La}$ quimioterapia es el pilar de tratamiento en fases avanzadas. El protocolo SMILE (dexametasona, metrotexato, ifosfamida, L- asparginasa y etopósido) es el más empleado como quimioterapia por su alta eficacia en etapas tempranas de la enfermedad pero presenta alta toxicidad, por lo que su beneficio es a corto plazo. ${ }^{21-24}$ El régimen PGEMOX (pegaspargasa, gemcitabina, oxaliplatino), antes conocido como GELOX (gemcitabina, L-asparginasa, oxaliplatino), seguido de radioterapia en pacientes con LNKTE en estadíos IE y IIE demostró beneficio a largo plazo y buen perfil de seguridad por su baja toxicidad; y, el DDGP (dexametasona, cisplatino, gemcitabina y pegaspargasa) que demostró mejor tolerancia y mayor efectividad en LNKTE avanzados recién diagnosticados respecto al SMILE. ${ }^{25,26}$ Por lo tanto, se recomienda radioterapia más quimioterapia basada en asparginasa en fases tempranas; y, quimioterapia basada en asparginasa con o sin radioterapia aunado a transplante autólogo de células madre o inmunoterapia. ${ }^{27}$ Nuestro paciente recibió tres sesiones de quimioterapia SMILE sin radioterapia.

El pronóstico está relacionado con la ubicación y el estadio de la enfermedad en el momento del diagnóstico, inicio tardío de tratamiento y la toxicidad del mismo., ${ }^{4,922}$ En general, el pronóstico del LNKTE es pobre. Se reportó que la supervivencia a 5 años en pacientes con enfermedad nasal fue $54 \%$ en contraste con los pacientes de enfermedad extranasal 34\% ${ }^{7,8,24}$ En una serie de casos de LNKTE con compromiso ocular, se describe una mortalidad de 77,8\%, teniendo peor sobrevida los LNKTE ocular primario, 5.1 \pm 6.5 meses, en comparación con LNKTE nasales con extensión ocular, 56.5 \pm 87.7 meses. ${ }^{5}$ Otros factores de mal pronóstico son los niveles de VEB en el plasma o la médula ósea y la toxicidad del tratamiento, por ello los pacientes deben estratificarse de manera que la intensidad del tratamiento se pueda modificar de acuerdo con el riesgo individual de cada paciente. ${ }^{11,22}$ Existen varios modelos como el Índice de pronóstico internacional (IPI), el Índice de pronóstico de Corea (KPI) y el Índice de pronóstico del linfoma asesino natural (PINK)que fueron validados para pacientes con linfoma de células Natural Killer/T (LNKT), de los cuales la enfermedad en estadio III o IV, el compromiso de ganglios linfáticos distales y la enfermedad no nasal se asocian significativamente con la supervivencia. ${ }^{23}$ El índice de riesgo revisado por normograma (NRI) considera la edad mayor de 60 años, deshidrogenasa láctica (DHL) elevada, entre otros de mal pronóstico. ${ }^{23} \mathrm{El}$ caso clínico que presentamos presentó un tiempo de supervivencia de 10 meses desde el inicio de los síntomas hasta su fallecimiento, con factores de mal pronóstico como el LNKT en región ocular periorbitaria, los niveles elevados DHL, el compromiso probable de piel y la terapia SMILE en un paciente con falla hepática y síntomas B establecidos.

En conclusión, el diagnóstico del LNKTE periorbitario se sugiere ante la presencia de celulitis palpebral que evoluciona a lesiones necróticas, costrosas y ulceradas refractarias a tratamiento antibiótico con resultados de biopsia repetitivos no concluyentes; pueden presentarse acompañados de falla hepática aguda.

\section{REFERENCIAS BIBLIOGRÁFICAS}

1. Saavedra Ramírez J, D: Linfoma Nasal de Células T/Natural Killer Extranodal Refractario Mal Diagnosticado, Tratado de Manera Exitosa: Informe de Caso. Case Rep Oncol. 2017;10:10921097. doi: 10.1159/000484583

2. Meel R, Dhiman R, Wadhwani M, Kashyap S, Sharma S, Gogia A: Isolated Extranodal Natural Killer T-Cell Lymphoma of the Orbit in a Young Patient: Complete Regression with the SMILE Regimen. Ocul Oncol Pathol. 2017;3:45-48. doi: 10.1159/000449227

3. Tse E, Kwong Y-L. The diagnosis and management of NK/T-cell lymphomas. J Hematol Oncol. 2017 Apr;10(1):85. Available from: https://pubmed.ncbi.nlm.nih.gov/28410601/

4. Jiménez-Pérez JC, Yoon MK. Natural Killer T-Cell Lymphoma of the Orbit: An Evidence-Based Approach. Semin Ophthalmol. 2017;32(1):116-24. Available from: https://pubmed.ncbi.nlm.nih. gov/27740876

5. Lee GI, Kim YD, Young SM, Shin S, Woo KI. Clinical characteristics and treatment outcomes of natural killer/T-cell lymphoma involving the ocular adnexa. Br J Ophthalmol. 2019 Feb;103(2):269-273. doi: 10.1136/bjophthalmol-2017-311704. Epub 2018 Apr 29. PMID: 29706604.

6. Wolkow N, Jakobiec FA, Habib LA, Freitag SK. Orbital Nasal-Type Extranodal Natural Killer/T-Cell Lymphoma: An Ongoing Diagnostic Challenge Further Confounded by Small-Cell Predominance. Ophthal Plast Reconstr Surg. 2019;35(5):478-83. Available from: https://europepmc.org/article/med/30865070

7. Tang GM, Chang TC, Tu X, Zhou GY, Liu ZZ. A Rapidly Progressing Fatal Case of Natural Killer/T-Cell Lymphoma Presenting as Orbital Inflammation. Chin Med J (Engl). 2018 Aug 20;131(16):20132014. doi: 10.4103/0366-6999.238146. PMID: 30082537; PMCID: PMC6085863.

8. Zuhaimy H, Aziz HA, Vasudevan S, Hui Hui S. Extranodal natural killer/T-cell lymphoma presenting as orbital cellulitis. GMS Ophthalmol Cases. 2017 Feb 6;7:Doc04. doi: 10.3205/oc000055. PMID: 28194321; PMCID: PMC5296617.

9. Zhang F, Duan X, Liu K. A case report of an extranodal NK/T-cell lymphoma nasal type, occurring primarily in eyes with masquerade syndrome. Medicine (Baltimore). 2019;98(11):e14836.

10. Bi Y, Wang S, Jiang Q, Yifei Y, Xiaoqiu L, Xiongzeng Z. Ocular Natural Killer-T Cell Lymphoma Clinicopathological analysis. Chinese J Ophthalmol. 2019;55(5):374-80. Available from: http://www.wanfangdata.com.cn/details/detail.do?_type= perio\&id=zhyk201905013

11. Yamaguchi M, Suzuki R. Treatment of extranodal NK/T cell lymphoma, nasal type. UpToDate, Inc. 2020 [cited 2020 Aug 31]. p. 1-34. Available from: https:/www.uptodate.com/contents/ treatment-of-extranodal-nk-t-cell-lymphoma-nasal-type/print Marzouki-Zerouali A, Charbit L, Mitcov M, Violon F, Schmutz

12. JL, Moawad S, et al. Extra-nodal NK/T-cell lymphoma, nasal-type, revealed by cutaneous and ocular involvement. Ann Dermatol Venereol. 2019;146(10):626-33. Available from: https://doi. org/10.1016/j.annder.2019.01.027

13. Ballinas-Aquino J, Arenas R, Vega-Memije ME, ToussaintCaire S. Linfoma NK nasal en adolescente. Dermatología Cosmética, Médica y Quirúrgica. 2016;14(2):131-6. Available from: https://www.medigraphic.com/pdfs/cosmetica/dcm-2016/dcm162g. pdf

14. Chan JY, Ng AYJ, Cheng CL, Nairismägi ML, Venkatesh B, Cheah $\mathrm{DMZ}$, et al. Whole exome sequencing identifies recessive germline mutations in FAM160A1 in familial NK/T cell lymphoma. Blood Cancer J. 2018 Nov 12;8(11):111. doi: 10.1038/s41408-018-01495. PMID: 30420729 ; PMCID: PMC6232147.

15. Copy

16. Cerhan JR, Slager SL. Familial predisposition and genetic risk factors for lymphoma. Blood. 2015;126(20):2265-73. Available 
from: https://pubmed.ncbi.nlm.nih.gov/26405224/

17. Al Omari A, Al Zoubi F, Alsalem MM, Al-Salem KM, Mohidat H. Primary sino-orbital peripheral t-cell lymphoma presenting as unilateral periorbital swelling: A case report. Int J Ophthalmol. 2018;11(11):1881-3. Available from: https://www.ncbi.nlm.nih. gov/pmc/articles/PMC6232323/

18. Björnsson ES. Drug-induced liver injury due to antibiotics. Scand J Gastroenterol. 2017;52(6-7):617-23. Available from: http://dx.doi. org/10.1080/00365521.2017.1291719

19. Shah I, Vyas N, Reynolds JA. Natural Killer-Like T-cell lymphoma: A rare cause of acute liver failure. ACG Case Reports J. 2019;6(7):e00145.

20. Matsuo T, Tanaka T, Ichimura K, Meguri Y. Intraocular relapse with hypopyon and retinal infiltrates after chemotherapy and peripheral blood stem cell transplantation for extranodal NK/T-cell lymphoma. $J$ Clin Exp Hematop. 2015;55(3):157-61. Available from: https:// europepmc.org/article/med/26763364

21. Al Shawabkeh MA, Al Sulaiti M, Al Sa'ey H, Ganesan S. Nasal type extranodal natural killer/T $(\mathrm{NK} / \mathrm{T})$ cell lymphoma presenting as periorbital cellulitis: A case report. Am J Case Rep. 2016 Dec;17:934-8. Available from: https:/www.ncbi.nlm.nih.gov/pmc/ articles/PMC5153321/

22. Chaudhary RK, Bhatt VR, Vose JM. Management of extranodal natural killer/T-cell lymphoma, nasal type. Clin Lymphoma, Myeloma Leuk. 2015;15(5):245-52. Available from: http://dx.doi. org/10.1016/j.clml.2014.12.014

23. Chen SY, Yang Y, Qi SN, Wang Y, Hu C, He X, et al. Validation of nomogram-revised risk index and comparison with other models for extranodal nasal-type NK/T-cell lymphoma in the modern chemotherapy era: indication for prognostication and clinical decision-making. Leukemia. 2021 Jan;35(1):130-142. doi: 10.1038/ s41375-020-0791-3. Epub 2020 Mar 9. PMID: 32152465; PMCID: PMC7787971.
24. Fox CP, Civallero M, Ko YH, Manni M, Skrypets T, Pileri S, et al. Survival outcomes of patients with extranodal natural-killer T-cell lymphoma: a prospective cohort study from the international T-cell Project. Lancet Haematol. 2020 Apr;7(4):e284-e294. doi: 10.1016/S2352-3026(19)30283-2. Epub 2020 Feb 24. PMID: 32105608 .

25. Li X, Cui Y, Sun Z, et al. DDGP versus SMILE in Newly Diagnosed Advanced Natural Killer/T-Cell Lymphoma: A Randomized Controlled, Multicenter, Open-label Study in China. Clin Cancer Res. 2016;22(21):5223-5228. doi:10.1158/10780432.CCR-16-0153

26. Wang L, Wang ZH, Chen XQ, Wang KF, Huang HQ, Xia ZJ. Firstline combination of GELOX followed by radiation therapy for patients with stage IE/IIE ENKTL: An updated analysis with long-term follow-up. Oncol Lett. 2015;10(2):1036-1040. doi:10.3892/ol.2015.3327

27. Wang L, Wang JW. Extranodal natural-killer T-cell lymphoma: experience from China. Lancet Haematol. 2020 Jun;7(6):e441. doi: 10.1016/S2352-3026(20)30103-4. PMID: 32470431.

\section{CORRESPONDENCIA \\ Dayana Carolina Gil-Clares \\ crousy_sum4I@hotmail.com}

Fecha de recepción: 25-10-2021.

Fecha de aceptación: 30-II-202I.

Conflicto de interés: ninguno, según los autores.

Financiamiento: por los autores. 